\title{
Annotation Tool for Enhancing E-Learning Courses
}

\author{
Bernardo Pereira Nunes ${ }^{1,2,3}$, Ricardo Kawase ${ }^{2}$, Stefan Dietze ${ }^{2}$, \\ Gilda Helena Bernardino de Campos ${ }^{3,4}$, and Wolfgang Nejdl ${ }^{2}$ \\ ${ }^{1}$ Department of Informatics - PUC-Rio - Rio de Janeiro, RJ, Brazil \\ bnunes@inf.puc-rio.br \\ ${ }^{2}$ L3S Research Center, Leibniz University Hannover, Germany \\ \{nunes, kawase, dietze, nejdl\}@13s.de \\ ${ }^{3}$ Central Coordination for Distance Learning - PUC-Rio - Rio de Janeiro, RJ - Brazil \\ \{bernardo,gilda\}@ccead.puc-rio.br \\ ${ }^{4}$ Education Department - PUC-Rio - Rio de Janeiro, RJ, Brazil \\ gilda@ccead.puc-rio.br
}

\begin{abstract}
One of the most popular forms of learning is through reading and for years we have used hard copy documents as the main material to learn. With the advent of the Internet and the fast development of new technologies, new tools have been developed to assist the learning process. However, reading is still the main learning method that is an individual activity. In this paper we propose a highlighting tool that enables the reading and learning process to become a collaborative and shared activity. In other words, the highlighting tool supports the so-called active-reading, a well-known and efficient means of learning. The highlighting tool brings to the digital environment the same metaphor of the traditional highlight marker and puts it in a social context. It enables users to emphasize certain portions of digital learning objects. Furthermore, it provides students, tutors, course coordinators and educational institutions new possibilities in the teaching and learning process. In this work we expose the first quantitative and qualitative results regarding the use of the highlight tool by over 750 students through 8 weeks of courses.
\end{abstract}

Keywords: e-Learning, Active-Reading, Online-Annotations, Evaluation, User Feedback.

\section{Introduction}

In the last decade we have witnessed an accelerated development of technologies that led us to create new forms of communication, learning situations and social skills. This advance in technology also stimulated a rapid growth of information and made a huge amount of information available anytime and anywhere. A flood of information is delivered every minute, making us to read more frequently than previous generations. However, the cognitive strategies that involve the learning process have not changed so fast. Still today, the so-called active reading [1] is the most prominent mean for learning and for stimulating critical thinking. Active reading involves mainly the actions of reading and annotating.

Annotating hard copy documents is a natural activity that involves direct interaction with the document and that is known to support understanding and memorization [2]. 
The term annotation comprises several techniques such as underline, circle or highlight important or confusing keywords and phrases; or writing notes to summarize or raise questions found in the text.

Nowadays, most of the queries with the purpose of learning are conducted on reference Web sites like Wikipedia as well as the debates around it, through the means of discussion forums, social networks or even e-mails that shifted the learning process from a paper-based activity and solitary task to a Web-based activity [5] and collaborative task.

However, due to limitations of the annotations tools on the Web, learners end up with a large collection of scattered digital resources. In most cases, annotations are out of context, written down on a piece of paper, on a separated digital document or e-mail, which impairs the information retrieval and the learning process.

The problem identified here is twofold. First, learners have a problem in contextualizing the annotations of a given online document. Second, the natural annotation activities are not supported in the Web. To overcome these problems we proposed an online annotation tool for online courses. We built our annotation tool upon the expertise learnt from the many pros and cons found in predecessor tools (see Section 2). Our annotation tool provides learners means to highlight any portion of the learning material available online. We decided for a minimalistic approach where users can intuitively make use of the tool, just by doing the same they would do when reading a hard copy document.

In addition to the highlighting, we provide two possible semantics for an annotation: confusing or important. In this way learners can not only better guide learning process, but also provide valuable feedback to the contents' authors. We assume that the contents' authors are constantly working with the students as a more capable pair. Thus, from the semantic-annotation done by students, the tutors can track students' development during the course.

In this paper we describe the technologies involved in the deployment of the tool applied on the online courses. Additionally, we present quantitative results of usage from a community of over 750 learners subscribed to the courses where the tool was available, together with qualitative results collected from a questionnaire.

The remainder of this paper is organized as follows. In the next Section, we review related work on past experiences and studies regarding online annotations. Section 3 describes the methodology involved in the online courses available in our system and Section 4 explains in details the usage of the highlight tool. In Section [5, we briefly describe the metrics used to collect students' feedback followed by a quantitative and qualitative results in Section 6 Finally, we present our conclusions and future directions in Section7

\section{Related Work}

In our work we define an annotation as any additional content that is directly attached to a resource and that adds some implicit or explicit information in many different forms [7]. This definition is in line with the definitions set forth by MacMullen [10] and Marshall [11].

Annotations are a common practice usually accompanying a reading task. They serve for different purposes. The most frequent annotations are used for signaling, supporting 
memorization and interpretation or for triggering reflection. Annotations also occur in many different forms: highlights, circles, symbols, writings, to name but a few. Each form of annotation is embedded with a certain use. Highlighting for example is mostly used to emphasize the importance of a certain part of the document. The same metaphor holds for encircling or underlining text. On the other hand, a strikethrough indicates that something is wrong, misplaced or not relevant. Also, to create relations between elements, lines and arrow-signals are used. In this work we focus on the highlight annotation metaphor which goals are to emphasize a certain portion of learning objects and classify it as important or confusing.

Vannevar Bush in the Memex [4] has long ago envisioned the benefits of electronic and automatic annotations. By relating all documents that users have read and attaching their annotations to these documents, individuals could organize and refind information resources in an associative manner, together with any earlier annotations. Although some Hypertext systems provide rich forms of annotations with categories, directions and multi-links, supporting associative trails, today's Web does not provide such functionalities. Information consumers have limited possibilities for writing and sharing comments on regular Web pages. As a result, a lot of time and effort is wasted when users try to comprehend the different formats of how people comment online resources. In the end, the work around solution is sending comments via e-mail [12].

In order to provide an unified standard for online annotations, as an extension of the open hypermedia Arakne Environment [3], the Fluid Annotations projects [13] deploys an online annotation system that supports in-context annotations. Their work focused on understanding the best metaphors to present digital annotations in terms of visual cues, regarding interactions, accommodation and animated transactions. Their work provides valuable feedback to the community for implementing usability and manipulation of annotations. Their main approach consists in accommodate annotations in between lines. Differently, we believe that disrupting the original information layout turns out to be more disruptive than beneficial.

Diigo is a more complete and commercial annotation tool. Diigo provides a toolbar with which users can highlight text or attach 'inline sticky notes' to Web pages. Despite the wealth of features, Diigo cannot boost a big user population. According to online user comments, this is due to both usability issues and the fact that all annotations are public by default. For a collaborative setup, reliability on the collaborators is a major issue for the beneficial outcomes of using the tool. Thus, a rather limited and trustful network of users is the best audience for a collaborative annotation tool.

In a past work, we have analyzed readers' annotation behavior during the learning process. In this way, we could fully comprehend the desired annotation features needed on the web. We conducted a field-study examining the paper-based annotations of 22 $\mathrm{PhD}$ students and pos-Docs in their own work environment [6]. For each participant, we looked at the last 3 research papers or articles that they have printed and read. In total we have collected 66 articles, covering a total of 591 pages of text. We found 1778 annotations and an average of 3.08 annotations per page. Out of these 1778 annotations, over $81 \%$ of them were simply highlights.

In addition to that, we have previously developed an in-context annotation tool for the broad audience, namely SpreadCrumbs [8]. Similar to Diigo the tool provides a toolbar 
that allows users to annotate with a floating sticky note on any Web page. Additionally, users were provided with searching and sharing functionalities within their social networks with great privacy control. In an oriented user study we have evaluated the usability of the tool, and most important, the benefits on online annotations in the tasks of refinding information [9]. The outcomes suggested that users of the annotation tool could refind information over two times faster. Furthermore, the usage of the browsers' 'find' functionality (CTRL+F) drops from an average of $53.5 \%$ to only $17.2 \%$.

Despite the benefits of the cited tools, none has reached a great audience. We believe that the excess of functionalities given by these tools is the main responsible for this failure. This failure increases the time of the learning process of the tools, and since the benefits are not perceived in a short-term use, users are most likely to abandon it. Also the inexistence of a smaller and trustful network with common interests imposes a barrier for the catch up of collaboration and mutual benefits of exchanging annotations. Considering these past experiences, we implemented an online highlight tool that (a) provides students a way to make their own annotations; (b) that allows the teachers to assess students' interaction in the content; and (c) that allows the contents' authors to improve and match the content of the course as students highlight the text.

\section{Online Courses}

The distance course 'Technology applied in Education' is designed for postgraduate students who wish to achieve literacy not only in information technology, but also to deepen the knowledge of it in the classroom. The course is aimed to those who are teachers in the educational public network in Brazil and aims to generate knowledge, promote teacher development and educational reform.

The first two editions of this course were held in 2006-2007 and 2009-2010 and resulted in the specialization of over 6000 teachers, distributed throughout Brazil. Although the main structure of the course is kept the same, each version of the course incorporates new tools and means of communication available, in order to suit current needs and prepare teachers to use and create new learning situations in their future lectures.

The 'Technology applied in Education' course is available all over Brazil and in the current version has over 750 subscribed students. Along with, the course has over 50 tutors that are responsible for monitoring, evaluating and teaching through our Learning Management System (LMS). Each tutor has a group of maximum 30 students. The course is delivered through online lectures, discussion forums, Web seminars and practical projects that support learning by doing.

Accordingly, we have deployed, in each edition of this specific course, several tools that can help students and teachers in the learning process. In this manner, here, we introduce the 'highlight tool', a simple yet powerful annotation tool, that has demonstrated a huge potential to improve and facilitate the learning process and course management.

For instance, the highlight tool has been demonstrated to be very useful to support the creation of courses focused on individual needs. Since the annotations are available to tutors, they can provide to each student additional materials or even create discussion 


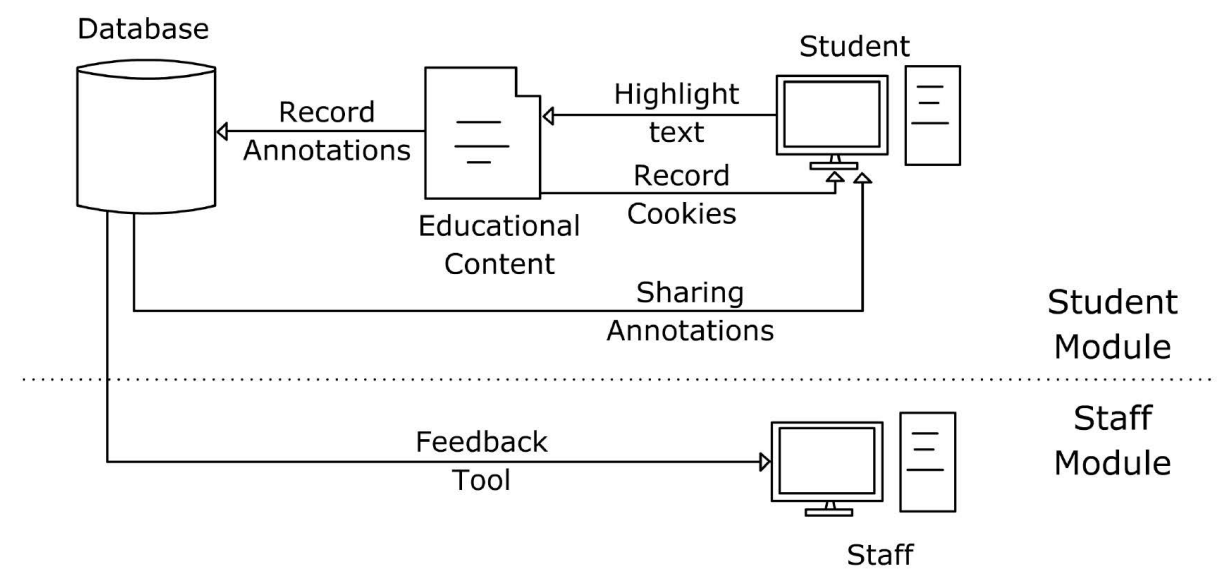

Fig. 1. The workflow shows the annotation process realized by a student. The annotations are stored in the LMS database and locally via cookies. All the annotations are available for analysis in the Staff Module.

forums to solve unexpected misunderstandings. This kind of tool allows us to create a non-mass course, adapting the structure of each content to fit students' needs and reach the goals of each lecture.

\section{The Highlight Annotation Tool}

The highlight tool is a reformulation of the traditional highlight pen used to emphasize excerpt of texts in hard copy documents. Usually, this pen is used to highlight relevant passages of text or passages that must be reviewed and easily found later. However, regardless of how good the highlight is, it will still have its own limitations.

With the advent of the Internet and the development of new technologies, simple tools like highlight pen could be redesigned to aggregate new possibilities of use and overcome its original use. Obviously, the first main difference from the traditional highlight pen is the fact that highlighted texts can be shared over the Internet. Consequently, all annotations done by the readers of a book could be sent to the publisher, which could analyze it and, if it is the case, update the next edition of a book.

For example, we commonly find typing errors in books, so this tool could help to correct them. In the e-learning context, this tool is even more powerful because students can share annotations between them and the teachers. The tool is boosted with an administration view, where teachers can analyze all the annotations done by their students. In this manner, teachers can have an overview of the content from the point of view of the students.

Moreover, teachers can discuss topics that most students thought confusing or even give more information about topics that they annotated as interesting. With the traditional highlight pen it would be impossible due to the time one would take to verify each annotation done by each student in a hard copy document. Hence, the highlight pen improves the whole learning development by assisting both learners and teachers within their tasks. 
In the Figure1, we show the workflow of the highlight tool. The highlight tool consists in two main modules, Student Module and Staff Module. The Student Module is responsible for recording all the annotations done by a student. The process is triggered at the moment the student selects one of the available highlight pens (confusing or important). For matters of simplicity and usability, we adopted only the two semanticannotations types mentioned before. However, the tool can be customized to use different colors and semantic-annotations types. Furthermore, before start the use of these tools, we introduced a brief description of its usage in order to ensure their understanding about each semantic-annotation type. Once the annotation is done, the annotated area is recorded in the LMS database. A copy of the annotation is also stored in the users' computer via cookies.

The reason to record the annotations in the users' computer is because they can also read the document when not connected to the Internet. Although this is an online course and one must have Internet connection to participate, this decision was made because in many regions in Brazil the Internet connection is dial-up or over satellite, thus, many students prefer to download the document, read, make their annotations and later connect again and participate online. In this manner, we record students' interactions twice, one in our database and another in their computers (via browsers' cookies).

The Staff Module is responsible for presenting the staff members (tutor, contents' author, course coordinator) highlighted texts in the online content (see Figure 2). In addition to that, an interface presenting statistics by course and type of annotation (confusing or important) help staff members to create and improve learning situations that better fits the students' needs. The tool can be installed in any document available in the Learning Management System (LMS), where we record all the annotations done by each student.

\section{Evaluation}

In order to evaluate the Highlight tool, we collected quantitative usage statistics and qualitative feedback from the learners. To assess qualitative feedback we set up an online questionnaire with 17 questions. We distributed the questionnaire to all students subscribed in the online courses that had access to the highlight tool. The questionnaire was not mandatory and was completely anonymous. We divide the questionnaire in five different tiers of questions, namely: usage, satisfaction, application, collaboration and future use.

- Usage. The questions regarding usage collect feedback regarding the students' access frequency to the online courses, the usage of the highlight tool and revisitation to the annotations.

- Satisfaction. Satisfaction covers the students' personal feeling regarding the tool concerning utility of the tool, if it supported their studies and easiness of use.

- Application. The questions of the application tier collect feedback considering possible applications and activities to be done on top of the annotations. For example, 'Is it beneficial to provide extra material for the annotations that are marked as important/confusing?'. 


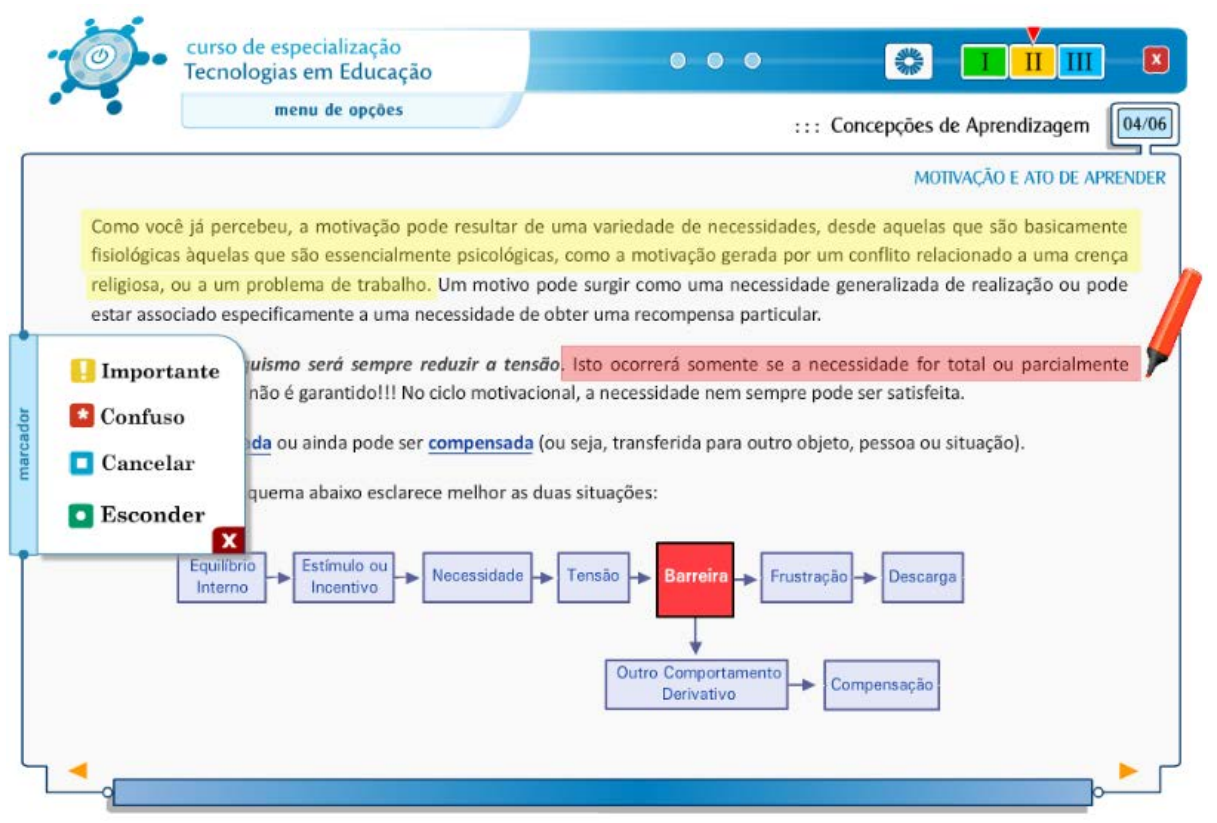

Fig. 2. Student Module. Example of annotations realized by a student. On top, an annotation marked as important(yellow) followed by an annotation marked as confusing(red).

- Collaboration. In terms of collaboration, although the tool did not provide any means for the students to exchange annotations, the questionnaire inquire them about their will to share and collaborate with other students. As one example, among the collaboration-related questions, we asked if they would like to have access to other students' annotations.

- Future Use. Finally the questions regarding future use address general opinions and inquire the students about their desires and plans to use the highlight tool in future courses.

For each question, the participants had to choose their agreement on a 5-point Likert scale.

\section{Results}

We collected the data from the first two courses where the tool was available. Each course consists of the main document - the one that can be annotated - together with other activities described in Section 3 and has a time-span of approximately eight weeks. We gathered the students' interaction during these 8 weeks. The first course consisted of a document containing 43 pages, while the second had 65 pages. In total we collected 279 annotations where $88 \%$ were marked as important. In Figure 3 we discriminate the annotations (important) by course and by page in each learning object. We did not find any correlation between the number of annotations marked as confusing and important. A thorough analysis of the portion of the most important-annotated 

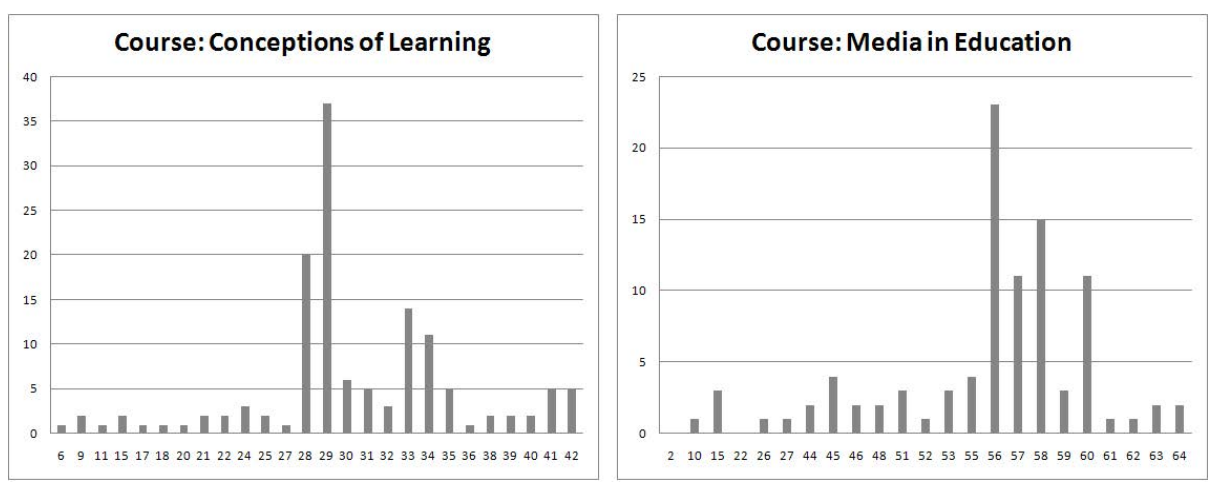

Fig. 3. Distribution of the annotations marked as important (y-axis) by page (x-axis) in the content of two distinct courses

pages, revealed us that the contents mainly contain definitions of concepts significant to the respective course.

In total, 132 students answered the questionnaire. In Table 1 we compile the answers distinguishing them by tear and agreement. Over $75 \%$ of the students that answered the questionnaire stated that they often (or very often) accessed the online content, however only $25 \%$ stated to use the highlight tool with the same frequency.

From the Satisfaction tier, over $77 \%$ agreed or strongly agreed that the highlight tool contributed to their learning process. Also, over $75 \%$ of the participants considered the tool straightforward to use.

Regarding the annotations and further activities that should be provided to the students, over $50 \%$ of the questionnaire participants agreed (or strongly agreed) that it is important to have further materials, discussion forums and other extra activities on the annotated topics. Peculiarly, the students considered on the same degree of agreement (without significant difference), that these activities would be helpful for both types of annotations, confusing or important.

Although the first goal of highlight tool is to provide students an individual method to support active-reading and refinding information, collaboration and communication also plays a major role in the learning process. Over $63 \%$ of the participants strongly agreed or agreed that collaborative features, as for example, sharing annotations and accessing other students' annotations, would definitely be beneficial during the learning process. By sharing annotations, or merely visualizing colleagues' highlights, students

Table 1. Results of the user experience questionnaire

\begin{tabular}{|c|c|c|c|}
\cline { 2 - 4 } \multicolumn{1}{c|}{} & Favor & Neutral & Against \\
\hline Usage & $54.23 \%$ & $23.81 \%$ & $21.96 \%$ \\
\hline Utility/Satisfaction & $66.14 \%$ & $20.11 \%$ & $13.76 \%$ \\
\hline Application & $51.72 \%$ & $25.79 \%$ & $22.49 \%$ \\
\hline Collaboration & $63.49 \%$ & $23.81 \%$ & $12.70 \%$ \\
\hline Future Use & $53.57 \%$ & $25.40 \%$ & $21.03 \%$ \\
\hline
\end{tabular}


can have a better overview on the importance of some portions of the learning objects, and also on the portions that raised more questions among her learning group. Shared annotations improve the individual learning and boost the online group discussion as well.

Finally, over 53\% of the participants would recommend the tool for colleagues and are also willing to use the tool in the next courses.

\section{Conclusion}

In this paper we present the highlight tool that aims to assist students in their learning process. As we demonstrated in this paper, once the annotations are recorded and available online, students, teachers/tutors, contents' authors and course coordinators can use this information to improve the content taught and the student's learning experience. The tool was deployed in an e-learning course with over 750 students that actively used it. Through the use of the tool the tutors could create new discussion topics to handle some students questions or to extend topics that was marked as interesting.

The annotations also contributed to improve the content available to the students. The contents' author reviewed the passages of the text that were very often marked as confusing or important. The texts that were marked as confusing are being reformulated. The texts that were marked as important are being expanded and in the next version of the course a complimentary material will be available for the students.

Finally, in the point of view of the course coordination or even of the institution, the tool is important to give feedback about the student needs, content quality and the continuity of the course. Through the use of this tool, the teachers can go beyond the group needs but also address individual needs of each student.

Through the feedback collected in our user evaluation, we conclude that the tool had a positive impact in the learning process and, moreover, students are willing to continue using the tool. As future works, we intend to expand the tool to recommend complimentary materials and enable collaborative features to enhance communication, facilitate social reading and to bring students closer, in order to improve the whole learning experience.

Acknowledgement. This research has been co-funded by the European Commission within the eContentplus targeted project OpenScout, grant ECP 2008 EDU 428016 (cf. http://www. openscout.net) and by CAPES (Process $n^{\circ}$ 9404-11-2).

\section{References}

1. Adler, M.J., Doren, C.V.: How to Read a Book. revised edn. Simon and Schuster, New York (1972)

2. Bernstein, M., Van Kleek, M., Karger, D., Schraefel, M.C.: Information scraps: How and why information eludes our personal information management tools. ACM Trans. Inf. Syst., 26(4), 24:1-24:46 (2008)

3. Bouvin, N.O.: Unifying strategies for web augmentation. In: Proceedings of the Tenth ACM Conference on Hypertext and Hypermedia: Returning to our Diverse Roots: Returning to our Diverse Roots, HYPERTEXT 1999, pp. 91-100. ACM, New York (1999) 
4. Bush, V.: As we may think. The Transatlantic Monthly (July 1945)

5. Chatti, M.A., Jarke, M.: The future of e-learning: a shift to knowledge networking and social software. Int. J. Knowledge and Learning 3(4/5), 404-420 (2007)

6. Kawase, R., Herder, E., Nejdl, W.: A Comparison of Paper-Based and Online Annotations in the Workplace. In: Cress, U., Dimitrova, V., Specht, M. (eds.) EC-TEL 2009. LNCS, vol. 5794, pp. 240-253. Springer, Heidelberg (2009)

7. Kawase, R., Herder, E., Papadakis, G., Nejdl, W.: In-Context Annotations for Refinding and Sharing. In: Filipe, J., Cordeiro, J. (eds.) WEBIST 2010. LNBIP, vol. 75, pp. 85-100. Springer, Heidelberg (2011)

8. Kawase, R., Nejdl, W.: A straightforward approach for online annotations: Spreadcrumbs enhancing and simplifying online collaboration. In: Filipe, J., Cordeiro, J. (eds.) WEBIST, pp. 407-410. INSTICC Press (2009)

9. Kawase, R., Papadakis, G., Herder, E., Nejdl, W.: The impact of bookmarks and annotations on refinding information. In: Proceedings of the 21st ACM Conference on Hypertext and Hypermedia, HT 2010, pp. 29-34. ACM, New York (2010)

10. Macmullen, W.J.: Annotation as process, thing, and knowledge: Multi-domain studies of structured data annotation. Technical report, in ASIST Annual Meeting (Charlotte, NC, 2005), ASIST (2005) (in review)

11. Marshall, C.C.: Annotation: from paper books to the digital library. In: DL 1997: Proceedings of the Second ACM International Conference on Digital Libraries, pp. 131-140. ACM Press, New York (1997)

12. Pirolli, P.: Information Foraging Theory: Adaptive Interaction with information. Oxford University Press, New York (2007)

13. Zellweger, P.T., Mangen, A., Newman, P.: Reading and writing fluid hypertext narratives. In: Proceedings of the Thirteenth ACM Conference on Hypertext and Hypermedia, HYPERTEXT 2002, pp. 45-54. ACM, New York (2002) 